\title{
As barreiras para a prática de atividade física estão associadas ao indicativo de sarcopenia de idosos?
}

\author{
Are barriers to physical activity associated with the indicative of sarcopenia in the \\ elderly?
}

Daniel Vicentini de Oliveira ${ }^{1 *}$, Naelly Renata Saraiva Pivetta ${ }^{2}$, Matheus Amarante do Nascimento ${ }^{3}$, Gabriel Lucas Morais Freire ${ }^{4}$, Sônia Maria Marques Gomes Bertolini ${ }^{1}$, José Roberto Andrade do Nascimento Júnior ${ }^{4}$

\begin{abstract}
${ }^{1}$ Doutor em Gerontologia. Centro Universitário de Maringá, Departamento de Pós-graduação em Promoção da Saúde de Maringá; ' Mestra em Gerontologia. Universidade Estadual de Campinas, Departamento de Gerontologia, Campinas/SP; ${ }^{3}$ Doutor em Educação física. Universidade Estadual do Paraná, Departamento de Educação Física, Paranavaí, Paraná; ${ }^{4}$ Mestrando em Educação Física. Universidade Federal do Vale do São Francisco, Departamento de Educação física de Petrolina/PE. ${ }^{5}$ Doutora em Ciências Morfológicas. Centro Universitário de Maringá,

Departamento de Pós-graduação em Promoção da Saúde, Maringá; ${ }^{6}$ Doutor em Educação física. Universidade Federal do Vale do São Francisco, Departamento de Educação física de Petrolina/PE.
\end{abstract}

\begin{abstract}
Resumo
Objetivo: investigar se as barreiras para a prática de atividade física estão associadas ao indicativo de sarcopenia de idosos. Metodologia: foram sujeitos 550 idosos com idades entre 60 e 89 anos, frequentadores das Academias da Terceira Idade do município de Maringá-PR. Como instrumentos foram utilizados o Questionário Internacional de Atividade Física (IPAQ), o questionário de percepção de barreiras para a prática de atividades físicas e o SARC-F. A análise de dados foi conduzida por meio dos testes de QuiQuadrado e o coeficiente de correlação de Spearman $(p<0,05)$. Resultados: as barreiras relacionadas à falta de clima adequado, falta de espaço disponível, falta de equipamento, tarefas domésticas, falta de companhia, mau humor, medo de lesionar-se, limitações físicas, dores leves ou mal-estar, falta de energia, falta de habilidades físicas, falta de conhecimento sobre AF, ambiente inseguro e falta de interesse foram significativamente $(p<0,05)$ mais frequentes entre os idosos com ausência de indicativo de sarcopenia. A correlação demonstrou associação inversamente proporcional entre a percepção de barreiras para a prática de AF e o indicativo de sarcopenia. Conclusão: o indicativo de sarcopenia não se mostrou um fator determinante para a apresentação de maiores barreiras de impedimento.
\end{abstract}

Palavras-chave: Idoso. Atividade motora. Exercício.

\begin{abstract}
Objecitve: investigated whether barriers to the practice of physical activity are associated with the indicative of sarcopenia in the elderly. Metodology: fifty-five elderly individuals between the ages of 60 and 89 years old, attending the Academies of the Third Age of the municipality of Maringá-PR. As instruments were used the International Physical Activity Questionnaire (IPAQ), the questionnaire of perception of barriers to the practice of physical activities and the SARC-F. Data analysis was conducted using the Chi-square test and the Spearman correlation coefficient $(p<0.05)$. Results: the results showed that barriers related to lack of adequate climate, lack of available space, lack of equipment, household chores, lack of company, moodiness, fear of injury, physical limitations, minor pain or malaise, lack of energy, lack of physical abilities, lack of knowledge about AF, insecure environment and lack of interest were significantly $(p<0.05)$ more frequent among the elderly with no indicative of sarcopenia. The correlation showed an inversely proportional association between the perception of barriers to the practice of PA and the indicative of sarcopenia. Conclusion: the indicative of sarcopenia was not a determining factor for the presentation of greater barriers of impediment.
\end{abstract}

Keywords: Elderly. Motor activity. Exercise.

\section{INTRODUÇÃO}

A relação entre atividade física $(\mathrm{AF})$, saúde e enveIhecimento vem sendo cada vez mais discutida e analisada mundialmente. Os avanços científicos na área de

Correspondente/Corresponding: ${ }^{*}$ Daniel Vicentini de Oliveira-End: Av. Londrina, 934, apartamento 1907, torre A. CEP: 87050-730. Maringá, Paraná - E-mail: d.vicentini@hotmail.com - Tel: gerontologia têm destacado que a manutenção de um estilo de vida ativo é um dos potenciais indicadores para a prevenção e o controle de comorbidades (FERREIRA et al., 2012; WALKER et al., 2011).

Com isso, a aquisição de hábitos alimentares saudáveis e a prática regular de exercícios físicos são considerados os pilares para um envelhecimento bem-sucedido (CORDEIRO et al., 2014; FERREIRA et al., 2012; PEREIRA 
et al., 2012). Nessa perspectiva, a literatura aponta evidências de que idosos envolvidos em programas de AF estão mais propensos a experimentar uma melhor função física e ter uma melhor expectativa de vida, quando comparados aos idosos inativos (RIBEIRO et al., 2015; SPOSITO; NERI; YASSUDA, 2016).

Com base nestes aspectos, observa-se que manter-se ativo fisicamente influencia positivamente as capacidades físicas dos idosos (LOPES et al., 2016; MILLÁN-CALENTI et al., 2010), enquanto que a inatividade física age como aceleradora do declínio físico dos mesmos (HIRVENSALO; LINTUNEN, 2011). Tal declínio pode decorrer, dentre outros aspectos, devido à redução da massa muscular que é capaz de ocasionar maior comprometimento funcional, perda de qualidade de vida, aumento do número de doenças, obesidade e mortalidade (VON BONSDORFF; RANTANEN, 2011). Além disso, há uma forte associação da redução da massa muscular com a diminuição da mobilidade e dos níveis de sarcopenia com o processo de envelhecimento (MONTER-FERNANDEZ; SERRA-REXACH, 2013; SANCHIS-GOMAR; GÓMEZ-CABRERA; VIÑA, 2011).

De acordo com o Consenso Europeu (EWGSOP2), a sarcopenia é classificada como uma doença muscular, do tipo insuficiência, enraizada em alterações musculares adversas que se acumulam num tempo de vida. Sua característica chave é a perda de massa e força na musculatura esquelética e seu diagnóstico baseia-se no desempenho físico (CRUZ-JENTOFT et al., 2018).

O déficit muscular proveniente dessa condição física está associado à redução de desempenho, dificuldades na realização dos movimentos básicos do cotidiano e comprometimento da prática de $A F$, interferindo negativamente no convívio social dos idosos (GENNUSO et al., 2013; GIANOUDIS; BAILEY; DALY, 2015; HAMER; STAMATAKIS, 2013; SEGUIN et al., 2012).

Além dos aspectos físicos, outros fatores tendem a reduzir os níveis de AF com o avanço da idade (CDCP, 2007). Estudos realizados em contextos diversos têm apresentado os fatores associados à alta ou baixa adesão aos exercícios (CORSEUIL et al., 2011), sendo que alguns destes influenciam de maneira positiva, sendo denominados "facilitadores" da prática de exercícios, enquanto outros influenciam de maneira negativa, sendo "barreiras" para a prática de exercícios (CASSOU et al., 2008).

Segundo estudo realizado por Sallis e Owen (1999), esses determinantes podem ser classificados em seis dimensões: demográficas e biológicas; psicológicas, cognitivas e emocionais; culturais e sociais; ambientais; características da AF e atributos comportamentais - o que demonstra a complexidade e diversidade dos aspectos que podem influenciar a prática de exercícios físicos. Uma pesquisa realizada no Estado de São Paulo evidenciou que existem diferenças entre as barreiras relatadas pelos residentes de cidades de grande e pequeno porte (ANDRADE et al., 2000).
Já Krug, Lopes e Mazo (2015), ao realizarem um estudo qualitativo com 30 idosas longevas e inativas fisicamente, encontraram como principiais barreiras a limitação física, a falta de disposição, o excesso de cuidado da família, o medo de quedas e o aumento da idade. A literatura aponta ainda que, para aqueles com alto nível socioeconômico, os fatores demográficos e biológicos são mais importantes, enquanto para os de baixo nível socioeconômico os fatores ambientais são preponderantes (CASSOU et al., 2008).

Sendo assim, compreender de maneira mais aprofundada a prática de AF e o risco de sarcopenia frente a essas barreiras experimentadas pode proporcionar um espaço informacional de grande utilidade no desenvolvimento de programas de intervenção no âmbito de saúde pública. Logo, o objetivo deste estudo foi investigar se as barreiras para a prática de AF estão associadas ao indicativo de sarcopenia de idosos.

\section{METODOLOGIA}

\section{Participantes}

A amostra foi escolhida de forma intencional e por conveniência. Foram sujeitos 550 idosos, de ambos os sexos, com idades entre 60 e 89 anos, frequentadores das Academias da Terceira Idade (ATI) do município de Maringá, estado do Paraná.

Devido à baixa prevalência de idosos usuários de algumas das ATI (informação relatada pela Secretaria de esportes e lazer do município) e/ou a precariedade ambiental e estrutural das mesmas, foram excluídas da coleta de dados 23 ATI. Portanto, do total de 57 ATI do município na época da coleta dos dados, foram pesquisadas 34 ATI.

Foram incluídos idosos de ambos os sexos, usuários de no mínimo uma das ATI pesquisadas, com capacidade de fala e audição preservadas, que permitissem a aplicação dos questionários. Idosos usuários de acessórios para a marcha, com déficits neurológicos e demenciais informados por cuidadores e/ou familiares no momento da coleta de dados, incapacitantes para a realização da pesquisa, foram excluídos. Foram excluídos também idosos com possíveis déficits cognitivos, avaliados por meio do Mini exame do estado mental (MEEM) (FOLSTEIN; FOLSTEIN; MCHUGH, 1975), de acordo com a escolaridade dos mesmos (BRUCKI et al., 2003).

\section{Instrumentos}

O nível de AF dos idosos foi avaliado por meio da versão curta do Questionário Internacional de Atividade Física (IPAQ). $O$ instrumento é composto por 7 questões abertas e suas informações permitem estimar o tempo despendido, por semana, em diferentes dimensões de AF (caminhadas e esforços físicos de intensidades moderada e vigorosa) e de inatividade física (posição sentada) (MATSUDO et al., 2001). 
A percepção de barreiras para a prática de AF foi avaliada pelo instrumento proposto por Martins e Petroski (2000). Para ser considerado uma "barreira percebida", cada fator devia ser citado pelo idoso como sendo "sempre" ou "quase sempre" um dificultante para a prática de AF.

Para avaliar os indicativos de sarcopenia foi aplicado o questionário SARC-F, teste desenvolvido por para diagnosticar a síndrome por meio de cinco componentes: força, assistência com caminhada a pé, subir de uma cadeira, subir escadas e histórico de quedas composto. As pontuações variam de 0 a 10 , com 0 a 2 pontos para cada componente sendo: SARC- $\mathrm{F}<4$ (sem indicativos de sarcopenia) e SARC-F $\geq 4$ (com indicativos de sarcopenia) ${ }^{26}$.

Procedimentos de coleta de dados

A pesquisa foi aprovada em seus aspectos éticos e metodológicos pelo Comitê de ética em pesquisa do Centro Universitário Metropolitano de Maringá (UNIFAMMA) sob protocolo no 2989.008/2018.

A equipe de pesquisa abordou os idosos nas ATI, em diferentes dias e horários. Foi entregue aos idosos um termo de consentimento livre e esclarecido, formalizando a participação voluntária na pesquisa. 0 tempo para aplicação das avaliações foi de aproximadamente 20 min por participante.

Análise dos dados

A análise dos dados foi realizada por meio do Software SPSS versão 22.0, mediante uma abordagem de estatística descritiva e inferencial. Foram utilizados frequência e percentual como medidas descritivas para as variáveis categóricas. A comparação das proporções foi conduzida por meio do teste Qui-Quadrado para variáveis dicotômicas e independentes. A correlação das barreiras para a prática de AF e o indicativo de sarcopenia foi verificada por meio do coeficiente de correlação de Spearman. Adotou-se o nível de 5\% de significância.

\section{RESULTADOS}

Dos 550 idosos avaliados, $62,7 \%$ eram do sexo feminino, com faixa etária entre 60 e 69 anos (54,9\%). Destes, $60,4 \%$ relataram ter companheiro, $62,8 \%$ dos idosos eram de etnia branca e $70,9 \%$ aposentados, com renda mensal de 1 a 2 salários mínimos (SM) $(55,4 \%)$. Observou-se que a maioria dos idosos não concluiu o ensino médio $(77,6 \%)$ e que a maioria dos participantes não apresentava indicativo de sarcopenia $(88,0 \%)$, de acordo com o SARC-F.

A análise das barreiras percebidas para a prática de AF evidenciou que as barreiras de Preocupação com a aparência $(79,6 \%)$, Falta de equipamento $(76,2 \%)$, Falta de espaço disponível (75,8\%), Falta de recursos financeiros $(74,9 \%)$ e Falta de incentivo da família/amigos $(74,2 \%)$ foram as principais barreiras que impedem ou dificultam os idosos de praticarem AF (Tabela 1).

Tabela 1-Barreiras para a prática de atividades físicas de idosos da cidade de Maringá, Paraná, Brasil.

\begin{tabular}{|c|c|c|c|c|}
\hline \multirow[t]{2}{*}{ Barreiras para a prática de AF } & \multicolumn{2}{|c|}{$\begin{array}{c}\text { Barreira não } \\
\text { percebida }\end{array}$} & \multicolumn{2}{|c|}{$\begin{array}{c}\text { Barreira } \\
\text { percebida }\end{array}$} \\
\hline & $\mathbf{F}$ & $\%$ & f & $\%$ \\
\hline Jornada de trabalho extensa & 192 & 34,9 & 358 & 65,1 \\
\hline Compromissos familiares & 341 & 62,0 & 209 & 38,0 \\
\hline Falta de clima adequado & 283 & 51,5 & 267 & 48,5 \\
\hline Falta de espaço disponível & 133 & 24,2 & 417 & 75,8 \\
\hline Falta de equipamento & 131 & 23,8 & 419 & 76,2 \\
\hline Tarefas domésticas & 336 & 61,1 & 214 & 38,9 \\
\hline Falta de companhia & 228 & 41,5 & 322 & 58,5 \\
\hline Falta de incentivo da família/amigos & 142 & 25,8 & 408 & 74,2 \\
\hline Falta de recursos financeiros & 138 & 25,1 & 412 & 74,9 \\
\hline Mau humor & 169 & 30,7 & 381 & 69,3 \\
\hline Medo de lesionar-se & 258 & 46,9 & 292 & 53,1 \\
\hline Limitações físicas & 247 & 44,9 & 303 & 55,1 \\
\hline Dores leves ou mal-estar & 296 & 53,8 & 254 & 46,2 \\
\hline Falta de energia & 254 & 46,2 & 296 & 53,8 \\
\hline Falta de habilidades físicas & 207 & 37,6 & 343 & 62,4 \\
\hline Falta de conhecimento sobre AF & 192 & 34,9 & 358 & 65,1 \\
\hline Ambiente insuficientemente seguro & 206 & 37,5 & 344 & 62,5 \\
\hline Preocupação com a aparência & 112 & 20,4 & 438 & 79,6 \\
\hline Falta de interesse & 167 & 30,4 & 383 & 69,6 \\
\hline
\end{tabular}

Nota. $A F=$ atividade física. $F=$ valores absolutos. $\%=$ valores relativos. Fonte: Os autores.

Ao estratificar as barreiras de acordo com o risco de sarcopenia (Tabela 2), as barreiras relacionadas à Falta de clima adequado, Falta de espaço disponível, Falta de equipamento, Tarefas domésticas, Falta de companhia, Mau humor, Medo de lesionar-se, Limitações físicas, Dores leves ou mal-estar, Falta de energia, Falta de habilidades físicas, Falta de conhecimento sobre AF, Ambiente inseguro e Falta de interesse foram significantemente $(p<$ $0,05)$ mais frequentes entre os idosos com ausência de indicativo de sarcopenia. 
Tabela 2 - Barreiras percebidas para a prática de atividades físicas entre idosos da cidade de Maringá, Paraná, Brasil, de acordo com o indicativo de sarcopenia.

\begin{tabular}{|c|c|c|c|c|c|c|}
\hline \multirow{3}{*}{$\begin{array}{c}\text { Barreiras para a } \\
\text { prática de AF }\end{array}$} & \multicolumn{4}{|c|}{$\begin{array}{l}\text { Indicativo de } \\
\text { sarcopenia }\end{array}$} & \multirow{3}{*}{$x^{2}$} & \\
\hline & \multicolumn{2}{|c|}{ Não } & \multicolumn{2}{|c|}{ Sim } & & \\
\hline & $\mathbf{F}$ & $\%$ & $f$ & $\%$ & & \\
\hline $\begin{array}{l}\text { Jornada de trabalho } \\
\text { extensa }\end{array}$ & 313 & 64,7 & 45 & 68,2 & 0,315 & 0,574 \\
\hline $\begin{array}{l}\text { Compromissos } \\
\text { familiares }\end{array}$ & 185 & 38,2 & 24 & 36,4 & 0,085 & 0,770 \\
\hline $\begin{array}{l}\text { Falta de clima } \\
\text { adequado }\end{array}$ & 246 & 50,8 & 21 & 31,8 & 8,401 & $0,004 *$ \\
\hline $\begin{array}{l}\text { Falta de espaço } \\
\text { disponível }\end{array}$ & 380 & 78,5 & 37 & 56,1 & 15,969 & $0,001^{*}$ \\
\hline $\begin{array}{l}\text { Falta de } \\
\text { equipamento }\end{array}$ & 379 & 78,3 & 40 & 60,6 & 10,028 & $0,002 *$ \\
\hline Tarefas domésticas & 198 & 40,9 & 16 & 24,2 & 6,787 & $0,009 *$ \\
\hline Falta de companhia & 293 & 60,5 & 29 & 43,9 & 6,593 & $0,010^{*}$ \\
\hline Falta de incentivo & 365 & 75,4 & 43 & 65,2 & 3,193 & 0,074 \\
\hline $\begin{array}{l}\text { Falta de recursos } \\
\text { financeiros }\end{array}$ & 369 & 76,2 & 43 & 65,2 & 3,799 & 0,051 \\
\hline Mau humor & 343 & 70,9 & 38 & 57,6 & 4,821 & $0,028^{*}$ \\
\hline Medo de lesionar-se & 271 & 56,0 & 21 & 31,8 & 13,628 & $0,001^{*}$ \\
\hline Limitações físicas & 286 & 59,1 & 17 & 25,8 & 26,084 & $0,001 *$ \\
\hline $\begin{array}{l}\text { Dores leves ou mal- } \\
\text { estar }\end{array}$ & 238 & 49,2 & 16 & 24,2 & 14,525 & $0,001^{*}$ \\
\hline Falta de energia & 277 & 57,2 & 19 & 28,8 & 18,906 & $0,001^{*}$ \\
\hline $\begin{array}{l}\text { Falta de habilidades } \\
\text { físicas }\end{array}$ & 314 & 64,9 & 29 & 43,9 & 10,847 & $0,001^{*}$ \\
\hline $\begin{array}{l}\text { Falta de conheci- } \\
\text { mento sobre AF }\end{array}$ & 329 & 68,0 & 29 & 43,9 & 14,767 & $0,001 *$ \\
\hline Ambiente inseguro & 314 & 64,9 & 30 & 45,5 & 9,352 & $0,002^{*}$ \\
\hline $\begin{array}{l}\text { Preocupação com a } \\
\text { aparência }\end{array}$ & 391 & 80,8 & 47 & 71,2 & 3,282 & 0,070 \\
\hline Falta de interesse & 345 & 71,3 & 38 & 57,6 & 5,160 & $0,023^{*}$ \\
\hline
\end{tabular}

Nota. ${ }^{*} p<0,05$ (Teste de Qui-quadrado). AF=atividade física. $F=$ valores absolutos. $\%$ = valores relativos.

Fonte: Os autores.

Ao analisar a correlação das barreiras para a prática de AF e o indicativo de sarcopenia dos idosos (Tabela 3), verificou-se que o indicativo de sarcopenia demonstrou correlação significante $(p<0,05)$ e negativa com as seguintes barreiras: Dores leves ou mal-estar $(r=-0,41)$, Limitações físicas $(r=-0,39)$, Medo de lesionar-se $(r=$ $-0,35)$, Falta de energia $(r=-0,35)$, Falta de habilidades físicas $(r=-0,32)$, Falta de companhia $(r=-0,30)$, Falta de conhecimento sobre AF $(r=-0,30)$, Ambiente inseguro $(r=-0,29)$, Falta de espaço disponível $(r=-0,27)$, Falta de clima adequado $(r=-0,25)$, Tarefas domésticas $(r=-$ $0,24)$, Falta de equipamento $(r=-0,20)$, Falta de recursos financeiros $(r=-0,20)$ e Mau humor $(r=-0,20)$. Estes achados indicam que existe uma associação inversamente proporcional entre a percepção de barreiras para a prática de AF e o indicativo de sarcopenia.
Tabela 3-Correlação das barreiras para a prática de atividades físicas e o indicativo de sarcopenia dos idosos da cidade de Maringá, Paraná, Brasil.

\begin{tabular}{ll}
\hline Barreiras para a prática de AF & Indicativo de sarcopenia \\
\hline Jornada de trabalho extensa & $-0,01$ \\
Compromissos familiares & $-0,10$ \\
Falta de clima adequado & $-0,25^{*}$ \\
Falta de espaço disponível & $-0,27^{*}$ \\
Falta de equipamento & $-0,20^{*}$ \\
Tarefas domésticas & $-0,24^{*}$ \\
Falta de companhia & $-0,30^{*}$ \\
Falta de incentivo & $-0,14$ \\
Falta de recursos financeiros & $-0,20^{*}$ \\
Mau humor & $-0,20^{*}$ \\
Medo de lesionar-se & $-0,35^{*}$ \\
Limitações físicas & $-0,39^{*}$ \\
Dores leves ou mal-estar & $-0,41^{*}$ \\
Falta de energia & $-0,35^{*}$ \\
Falta de habilidades físicas & $-0,32^{*}$ \\
Falta de conhecimento sobre AF & $-0,30^{*}$ \\
Ambiente inseguro & $-0,29^{*}$ \\
Preocupação com a aparência & $-0,15$ \\
Falta de interesse & $-0,11$ \\
\hline
\end{tabular}

Nota. ${ }^{*} p<0,05$ (Correlação de Spearman). AF = atividade física. $F=$ valores absolutos. $\%=$ valores relativos.

Fonte: Os autores.

\section{DISCUSSÃO}

Os achados deste estudo revelaram que as principais barreiras para a prática de AF apontadas pelos idosos de Maringá-PR foram referentes à preocupação com a aparência fisica, falta de equipamento e espaços disponíveis, falta de recurso financeiro, falta de incentivo da família/amigos.

Estes resultados diferem dos encontrados no estudo desenvolvido por Krug, Lopes e Mazo (2015), que verificaram em idosas longevas fisicamenre inativas de Florianópolis-SC, que as principiais barreiras para a prática de AF foram a limitação física, falta de disposição, excesso de cuidado da família, medo de quedas, bem como o aumento da idade. Já os facilitadores apontados pelas idosas foram referentes ao prazer pela prática, socialização e busca pela saúde.

Quanto às barreiras, especificamente, verifica-se que os aspectos físicos são os principais dificultantes na realização das atividades para pessoas idosas, uma vez que apresentam limitações funcionais que reduzem a realização de atividades de vida diária e também de AF. Para além disso, as questões motivacionais, tais como a 
disposição também implicam na ausência de envolvimento dos idosos nas práticas de atividades, aspectos estes que devem ser pesquisados de forma mais aprofundada com vistas a atender às demandas desta população.

Outra pesquisa com resultados contrários aos do presente estudo foi realizada por Ribeiro et al. (2015), que buscaram identificar os fatores para a não participação das idosas de Uberaba - MG em programas de AF. Os autores observaram como principais barreiras para o engajamento das idosas as questões relacionadas aos sintomas depressivos, prática insuficiente de AF no lazer, bem como a baixa autoeficácia percebida para a realização de AF moderadas e vigorosas. Conforme destacado, o fator referente aos sintomas depressivos pode resultar em reduzida participação social com tendência ao isolamento e desânimo, o que leva os idosos a evitarem as atividades realizadas em grupos, como os programas de AF.

Conforme reportado na literatura, os fatores determinantes que incidem sobre o interesse dos sujeitos para o envolvimento com a atividade/exercício físico podem ser contemplados a partir da análise das seis esferas: aspectos demográficos e biológicos; psicológicos, cognitivos e emocionais; culturais e sociais; ambientais; características da AF e atributos comportamentais (CORSEUIL et al., 2011).

Ao analisar os achados da presente pesquisa sob esta ótica, percebe-se uma predominância dos aspectos biológicos e psicológicos quando se considera a aparência física como o principal fator, seguida da importância dada aos aspectos ambientais (espaços, segurança, equipamentos). Diante desses aspectos, observa-se a complexidade e diversidade dos aspectos que podem influenciar as pessoas à prática de exercicios físicos, os quais vão muito além dos interesses pessoais ou condições fisicas.

Outro aspecto relevante observado nesta pesquisa evidenciou que os idosos não identificados com indicativo de sarcopenia apresentaram maiores percepções de barreiras para a prática de AF, prinicipalmente no que tange aos espaços fisicos, limitações e falta de habilidade, ausência de companhia e de conhecimentos sobre a importância da prática da AF para a saúde. Por meio desses achados pode-se inferir que, mesmo que o idoso não apresente condições de saúde que limitem suas funções físicas, a ausência de espaços adequados e seguros pode ser fundamental para que seu engajamento nos programas de atividades seja dificultado.

Estes achados são contrários à literatura que aponta que os idosos identificados com indicativo de sarcopenia apresentam baixa força muscular, redução do desempenho para a realização de atividades de vida diária e AF (BARBOSA-SILVA et al., 2016). Devido a estas características, pode-se inferir que, apesar das recomendações acerca da relevância da prática de exercícios para as limitações decorrentes da sarcopenia, muitos idosos tendem a evitar o contato com as atividades, devido à reduzida funcionalidade para a realização das mesmas. A partir dos resultados evidenciados no presente estudo, verifica-se a necessidade de investimentos em políticas de divulgação e orientações aos idosos das comunidades quanto aos benefícios da prática de atividade. Tais iniciativas podem partir das universidades que ofertam inúmeros projetos de AF para a população idosa, fomentando suas relações com a melhora na função cardiovascular, fisiológica, motivacional e também no combate à sarcopenia.

A presente investigação evidenciou que as relações negativas do indicativo de sarcopenia com as barreiras para prática de $A F$, indicam que quanto menor o indicativo de sarcopenia, maior as proporções de barreira encontradas para os idosos para a prática de AF. Estes aspectos podem indicar que não sãos os aspectos biológicos ou físicos que limitam o engajamento dos idosos de Maringá-PR nestas práticas, mas, as dimensões psicológicas (comportamentais) se mostram preponderantes para a não inserção dos mesmos.

Apesar das importantes contribuições deste estudo para a compreensão dos fatores associados ao risco de sarcopenia em idosos paranaenses, algumas limitações precisam ser destacadas. Primeiramente, pontuamos a utilização de somente um instrumento para avaliação de indicativo de sarcopenia por medida de auto relato, o que pode comprometer a real mensuração do déficit muscular dos idosos. Uma segunda limitação refere-se a não avaliação dos níveis de AF dos idosos participantes da presente pesquisa, o que não permite a verificação da associação com os níveis de indicativo de sarcopenia. Uma terceira limitação refere-se ao design do estudo transversal, que não permite a avaliação destas variáveis ao longo de determinado período para acompanhamento das reais condições funcionais dos idosos paranaenses. Diante disso, para futuras pesquisas sugere-se a mensuração dos níveis de AF junto às identificações das barreiras e facilitadores da prática de $\mathrm{AF}$ em idosos, mensuração dos níveis de indicativo de sarcopenia por protocolos específicos e acompanhamento destas avaliações ao longo do tempo, bem como a oferta de programas específicos para desenvolvimento da força muscular em idosos.

Os resultados revelaram que os fatores de aparência física, espaços/equipamentos e falta de incentivo para a prática ou de conhecimento sobre a importância da AF são as principais barreiras para a inserção dos idosos nos programas de exercícios. Verificou-se ainda que o indicativo de sarcopenia não se mostrou um fator determinante para a apresentação de maiores barreiras de impedimento.

Como implicações práticas esta pesquisa contribui para a compreensão dos indicadores referentes às barreiras para a pratica de AF para a população idosa paranaense com vistas à estruturação de programas de saúde e políticas públicas em prol da saúde que atendam as reais necessidades e aspirações dos idosos, criando espaços/ ambientes adequados e que fomentem a prática de AF como uma ferramenta para a prevenção das limitações decorrentes do processo natural de envelhecimento. 


\section{REFERÊNCIAS}

ANDRADE, E. L. et al. Barriers and motivational factors for physical activity adherence in elderly people in developing country. Med. Sci. Sports Exerc., Baltimore, v. 33, n. 7, p. 141, 2000.

BARBOSA-SILVA, T. G. et al. Enhancing SARC-F: Improving sarcopenia screening in the clinical practice. J. Am. Med. Dir. Assoc., Hagerstown, v. 17, n. 12 , p. $1136-1141,2016$.

BRUCKI, S. M. D et al. Sugestões para o uso do mini-exame do estado mental no Brasil. Arq. Neuro-Psiquiatr., São Paulo, v. 61, n. 3B, p. 777781, 2003.

CASSOU, A. C. et al. Barreiras para a atividade física em idosos: uma análise por grupos focais. J. Phys. Educ., [s.I], v. 19, n. 3, p. 353-360, 2008.

CORDEIRO, J. et al. Efeitos da atividade física na memória declarativa, capacidade funcional e qualidade de vida em idosos. Rev. Bras. Geriat. Gerontol., Rio de Janeiro,v. 17, n. 3, p. 541-552, 2014.

CENTERS FOR DISEASE CONTROL AND PREVENTION(CDCP). Prevalence of regular physical activity among adults-United States, 2001 and 2005. Morb. Mortal. Wkly. Rep., Atlanta, v. 56, n. 46, p. 1209-1212, 2007.

CORSEUIL, M. W. et al. Perception of environmental obstacles to commuting physical activity in Brazilian elderly. Prev. med., New York, v. 53, n. 4-5, p. 289-292, 2011.

CRUZ-JENTOFT, A. J. et al. Sarcopenia: revised European consensus on definition and diagnosis. Age ageing, London, v. 48, n. 1, p. 16-31, 2018.

FERREIRA, O. G. L. et al. Envejecimiento activo y su relación con la independencia funcional. Texto contexto-enferm., Santa Catarina, v. 21, n. 3, p. 513-518, 2012.

FOLSTEIN, M. F.; FOLSTEIN, S. E.; MCHUGH, P. R. "Mini-mental state": a practical method for grading the cognitive state of patients for the clinician. J. psychiatr. res., Oxford, v. 12, n. 3, p. 189-198, 1975.

GENNUSO, K. P. et al. Sedentary behavior, physical activity, and markers of health in older adults. Med. sci. sports exerc., Madison, v. 45, n. 8 , p. 1493, 2013.

GIANOUDIS, J.; BAILEY, C. A.; DALY, R. M. Associations between sedentary behaviour and body composition, muscle function and sarcopenia in community-dwelling older adults. Osteoporosis int., London, v. 26, n. 2, p. 571-579, 2015.

HAMER, M.; STAMATAKIS, E. Screen-based sedentary behavior, physical activity, and muscle strength in the English longitudinal study of ageing. PLos ONE, San Francisco, v. 8, n. 6, p. e66222, 2013.

HIRVENSALO, M.; LINTUNEN, T. Life-course perspective for physical activity and sports participation. Eur. Rev. Aging Phys. A, Germany, v. 8, n. 1, p. 13, 2011.

KRUG, R. de R.; LOPES, M. A.; MAZO, G. Z. Barreiras e facilitadores para a prática da atividade física de longevas inativas fisicamente. Rev. bras. med. esporte, São Paulo, v. 21, n. 1, p. 57-64, 2015.
LOPES, M. A. et al. Barreiras que influenciaram a não adoção de atividade física por longevas. Rev. Bras. Ciênc. Esporte, Campinas, v. 38, n. 1, p. 76-83, 2016.

MATSUDO, S. et al. Questinário internacional de atividade f1sica (IPAQ): estudo de validade e reprodutibilidade no Brasil. Rev. bras. ativ. fís. saúde, Londrina, p. 05-18, 2001.

MARTINS, M. de O.; PETROSKI, E. L. Mensuração da percepção de barreiras para a prática de atividades físicas: uma proposta de instrumento. Rev. bras. cineantropom. desempenho hum, Florianopólis, v. 2, n. 1, 2000.

MILLÁN-CALENTI, J. C. et al. Prevalence of functional disability in activities of daily living (ADL), instrumental activities of daily living (IADL) and associated factors, as predictors of morbidity and mortality. Arch. gerontol. geriatr., Amsterdam, v. 50, n. 3, p. 306-310, 2010.

MONTERO-FERNANDEZ, N.; SERRA-REXACH, J. A. Role of exercise on sarcopenia in the elderly. Eur. J. Phys. Rehabil. Med., Torino, v. 49, n. 1, p. 131-143, 2013.

PEREIRA, G. N. et al. Indicadores demográficos e socioeconômicos associados à incapacidade funcional em idosos. Cad. Saúde Pública, Rio de Janeiro, v. 28, p. 2035-2042, 2012.

RIBEIRO, D. K. de M. N. et al. Fatores contributivos para a independência funcional de idosos longevos. Rev. Esc. Enferm. USP, São Paulo, v. 49, n. 1, p. 89-96, 2015.

RIBEIRO, A. S. et al. Effect of resistance training on C-reactive protein, blood glucose and lipid profile in older women with differing levels of RT experience. Age, London, v. 37, n. 6, p. 109, 2015.

SALLIS, J. F.; OWEN, N. Physical activity and behavioral medicine. California: SAGE Publications, 1999. (Behavioral Medicine and Health Psychology Series).

SANCHIS-GOMAR, F.; GÓMEZ-CABRERA, M. C.; VIÑA, J. The loss of muscle mass and sarcopenia: non hormonal intervention. Exp. Gerontology, Oxford, v. 46, n. 12, p. 967-969, 2011.

SEGUIN, R. et al. Sedentary behavior and physical function decline in older women: findings from the Women's Health Initiative. J. aging res., France, v. 2012, 2012.

SPOSITO, G.; NERI, A. L.; YASSUDA, M. S. Atividades avançadas de vida diária (AAVDs) e o desempenho cognitivo em idosos residentes na comunidade: Dados do Estudo FIBRA Polo UNICAMP. Rev . Bras. Geriatr. Gerontol., Rio de Janeiro, v. 19, n. 1, p. 7-20, 2016.

VON BONSDORFF, M. B.; RANTANEN, T. Progression of functional limitations in relation to physical activity: a life course approach. Eur. Rev. Aging Phys. A, Germany, v. 8, n. 1, p. 23, 2011.

WALKER, D. K. et al. Exercise, amino acids and aging in the control of human muscle protein synthesis. Med. sci sports exerc., Madison, $v$. 43, n. 12, p. 2249, 2011.

Submetido em: $28 / 08 / 2019$

Aceito em: 30/01/2020 